\title{
Receptor-mediated selective impairment of insulin-like growth factor-1 activity in congenital disorders of glycosylation patients
}

\author{
Gursev S. Dhaunsi'
}

BACKGROUND: Congenital disorders of glycosylation (CDG) patients share a basic feature of protein hypoglycosylation. Activity of growth factors and their receptors, glycoproteins playing a pivotal role during child development, remains unexplored in CDG patients.

METHODS: Peripheral blood lymphocytes (PBL) isolated from 9 CDG patients and 12 healthy controls were cultured in the presence of fetal bovine serum (FBS), platelet-derived growth factor (PDGF), basic fibroblast growth factor (bFGF), and insulin-like growth factor-1 (IGF-1), and BrdU incorporation was measured. Levels of plasma IGF-1 and PBL IGF-1 receptor (IGF-1R) and its glycosylation were detected using immunoassay and western blot.

RESULTS: CDG patients showed significantly less $(P<0.01)$ serum-induced 5'-Bromo-2'-deoxyuridine (BrdU) incorporation in PBL than in controls. PDGF-/FGF-stimulated BrdU incorporation showed no difference in patients and controls, whereas IGF-1-induced DNA synthesis was significantly $(P<$ 0.01 less in patients. Plasma IGF-1 levels and PBL IGF-1 receptor protein were significantly $(P<0.01)$ reduced in patients as compared to controls. IGF-1 receptor in PBL of all CDG patients had significantly $(P<0.01)$ reduced carbohydrate content when compared with control.

CONCLUSIONS: These results show selective impairment of IGF-1-induced DNA synthesis in lymphocytes of CDG patients through decreased gene expression and hypoglycosylation of the IGF-1 receptor.

C ongenital disorders of glycosylation (CDG) are relatively a new group of rare autosomal recessive metabolic diseases with well-described clinical characteristics $(1,2)$. Though the clinical spectrum of CDG is continuing to evolve with reports from various parts of the world, major nervous system impairment and developmental delay are the key features of this multisystemic group of diseases (3). Though, a new nomenclature has been established for CDG due to tremendous increase in number of CDG reports about variations in clinical phenotypes/ genotypes, yet CDG is broadly classified into type Ix and TypeIIx until detection of a specific defect (4). A large incidence of autosomal recessive disorders in the Middle East is well known, however CDG have remained undiagnosed until recently. A number of CDG cases have now been reported from the Arab populations, and we have earlier described some distinct clinical features of a CDG type II case from Kuwait (5). A common metabolic defect of protein hypoglycosylation is a hallmark of all CDG types and basis for biochemical diagnosis of patients by isoelectric focusing of serum glycoproteins such as transferrin $(6,7)$. Although the underlying biochemical and molecular defects in various types of CDG are known, yet molecular mechanisms linked with pathogenesis of the disease have remained unexplored. Besides several other clinical abnormalities, developmental delay or growth retardation are commonly observed in CDG patients. During perinatal, neonatal, and postnatal periods of human development, growth factors such as growth hormone and insulin-like growth factor-1 (IGF-1) are known to play an important role and to determine the growth and function of various organs and tissues (8-10). Most plasma membrane and secretory proteins, including growth factors and their receptors, undergo post-translational modification of glycosylation, thus forming the glycoproteins that function as integral components of cellular systems such as mitogens, cytokines, bioactive cell surface receptors, immunoglobulins, and several enzymes (11). CDG patients have basic metabolic defect of deglycosyaltion/ nonglycosylation of proteins that could lead to abnormal biosynthesis and malfunction of some glycoprotein growth factors and/or their receptors. Therefore, we have examined the growth factor-stimulated mitogenic activity in lymphocytes of CDG patients in order to get some insights into possible role of growth factors and their receptors in pathogenesis of CDG.

\section{RESULTS}

Impairment of Growth Factor-Stimulated DNA Synthesis in CDG Patients

In order to examine the status of growth factor mitogenic activity in CDG patients, DNA synthesis was assessed in lymphocytes isolated from CDG patients and healthy control subjects. Figure 1 shows BrdU incorporation (an index of DNA synthesis) in lymphocytes stimulated with 5\% fetal bovine serum (FBS) in comparison to lymphocytes in 


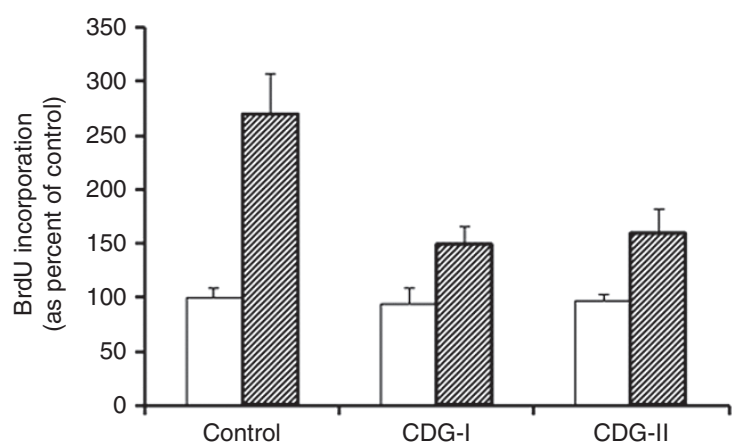

Figure 1. BrdU incorporation in lymphocytes from control subjects and, CDG-I and CDG-II patients in a serum-free medium (open bars) and in response to treatment with $5 \%$ fetal bovine serum (FBS) (shaded bars). Values are mean \pm SD of six assays. $P<0.01$ when CDG-I and CDG-II groups were compared with control FBS-stimulated lymphocytes.
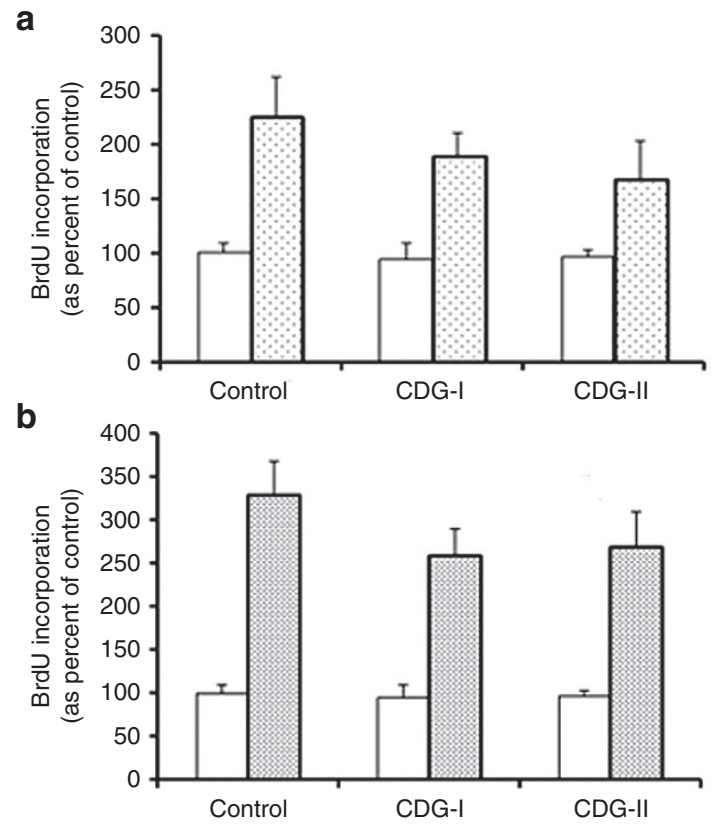

Figure 2. BrdU incorporation in lymphocytes from control subjects and CDG (types I and II) patients following $24 \mathrm{~h}$ stimulation with plateletderived growth factor (a) and basic fibroblast growth factor (b). Open bars represent cells treated with serum-free medium and shaded bars show growth factor-treated cells. Values are mean \pm SD of six determinations.

serum-free medium. When stimulated with 5\% FBS, peripheral blood lymphocytes (PBL) of CDG type- 1 as well as typeII patients were observed to have significantly $(P<0.05)$ lower BrdU incorporation (148 and 169\% of serum-free medium, respectively) compared to lymphocytes isolated from healthy counterparts $(269 \%$ of serum-free medium). To further assess the mitogenic activity of individual growth factors in CDG patients, lymphocytes were treated with $50 \mathrm{ng} / \mathrm{ml}$ of platelet-derived growth factor (PDGF) or bFGF, and as shown in Figure 2, BrdU incorporation into lymphocytes of CDG type-I and type-II patients was not markedly different from that into lymphocytes from healthy control subjects. Lymphocytes isolated from CDG patients (both type-I and type-II) were however, observed to have a significantly lower a
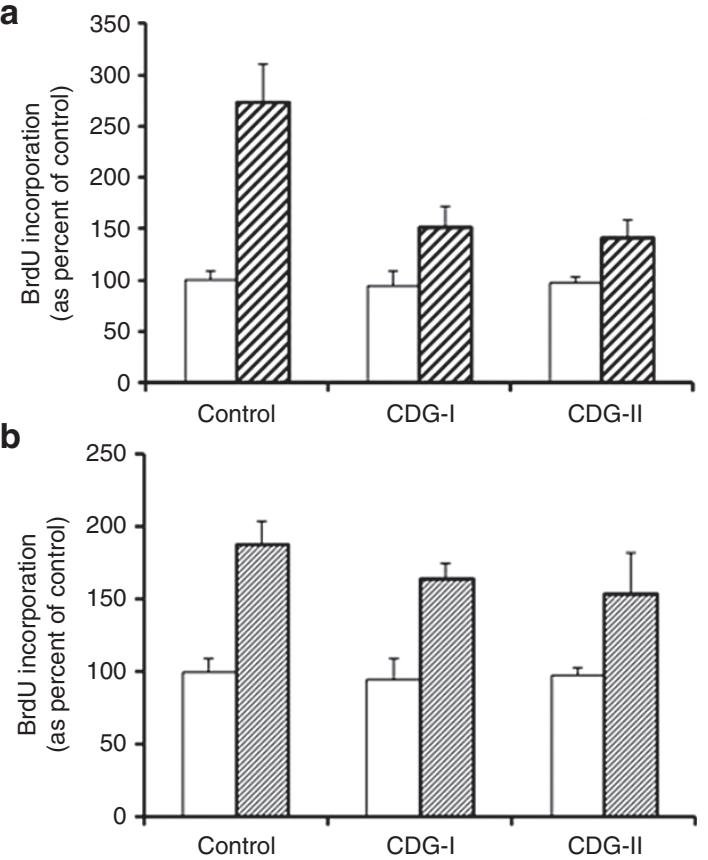

Figure 3. BrdU incorporation in lymphocytes from control subjects and CDG (types I and II) patients following $24 \mathrm{~h}$ stimulation with insulin-like growth factor-1 (IGF-1) (a) and insulin-transferrin-selenium (b). Open bars represent cells treated with serum-free medium and shaded bars show growth factor-treated cells. Values are mean \pm SD of six determinations. $P<0.01$ when CDG-I and CDG-II groups were compared with IGF-1stimulated control lymphocytes.

$(P<0.05)$ BrdU incorporation compared to lymphocytes of healthy controls when stimulated with $50 \mathrm{ng} / \mathrm{ml}$ of IGF-1 (Figure 3a). As shown in Figure 3b, insulin-transferrinselenium-induced DNA synthesis (BrdU incorporation) was similar in lymphocyte cultures of CDG patients as well as control subjects. It is worth mentioning that lymphocytes isolated from all patients showed impairment of FBS- and IGF-1-induced mitogenesis.

\section{IGF-1 Levels and IGF-1 Receptor Protein Expression in CDG Patients}

With regard to the observed impairment of IGF-1 mitogenic activity in lymphocytes of CDG patients, we measured plasma levels of IGF-1. Figure 4 shows that circulating levels of IGF-1 were lower in CDG patients when compared with healthy controls, however the results were significant for type-II patients. Western blot analysis of lymphocytes revealed that IGF-1 receptor protein was significantly decreased $(P<0.05)$ in CDG type-I patients but remained unaltered in CDG type-II patients when compared with control subjects (Figure 5).

\section{Glycosylation of IGF-1 Receptor Protein in CDG Patients}

To examine the glycosylation level of IGF-1 receptor in CDG patients, glycoprotein detection assay was performed on immunoprecipitated IGF-1 receptor protein and both CDG type-I as well as CDG type-II patients were observed to have remarkable deglycosylation of their lymphocytic IGF-1 R protein when compared with control subjects as depicted by 


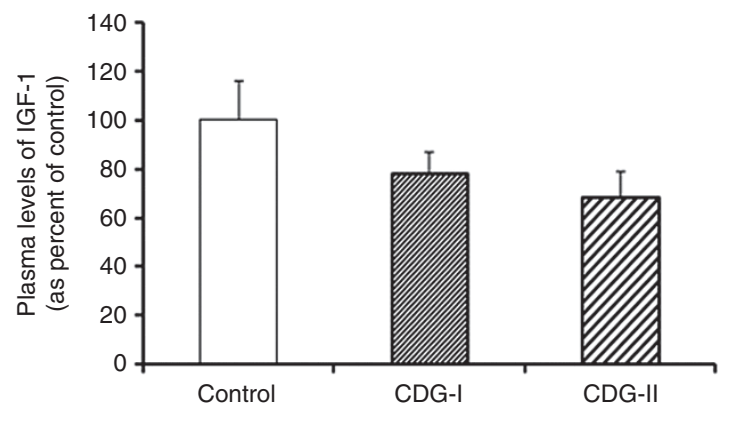

Figure 4. Plasma levels of insulin-like growth factor-1 in control subjects and, CDG I and CDG II patients. Values are mean \pm SD of six determinations. $P<0.01$ when CDG-II compared with control.

a

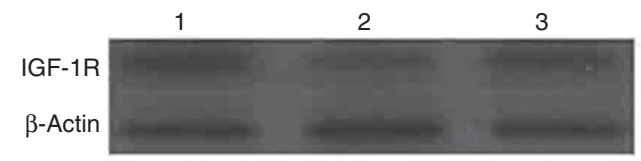

b

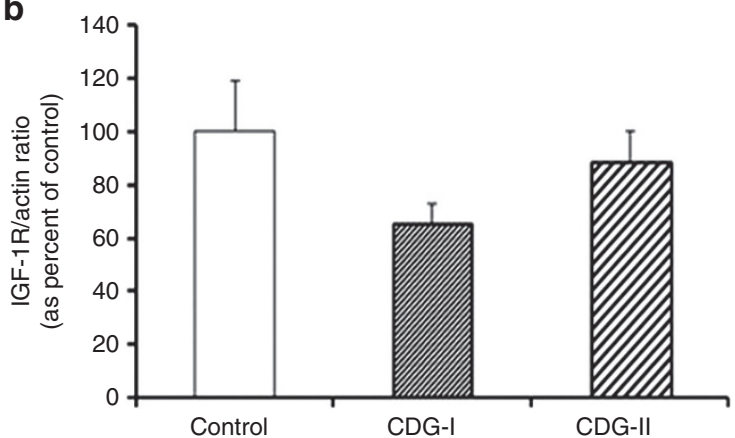

Figure 5. Western blot detection of the insulin-like growth factor-1 (IGF1)-receptor (IGF-1R) protein levels in lymphocytes of control subjects and CDG patients. Panel a shows the representative blots. Results shown in panel $\mathbf{b}$ are the ratio of IGF-1R protein and $\beta$-actin from six determinations. $P<0.01$ when CDG-I compared with control.

significantly decreased $(P<0.05)$ carbohydrate content of IGF-1 receptor in Figure 6.

\section{DISCUSSION}

CDG are inherited metabolic diseases with a wide range of clinical abnormalities and a common well-defined biochemical defect of protein deglycosylation. Though several studies on various aspects of CDG are reported on a regular basis, yet molecular mechanisms associated with pathogenesis of CDG have remained unexplored. This study provides some new insights into a possible role of growth factors, particularly IGF1 , in pathogenesis of CDG.

Oligosaccharides are covalently linked to proteins through enzymatic reactions during the formation of glycoproteins and inherited or acquired structural alteration(s) in growth factors and their receptors during protein glycosylation can affect various stages of human development $(11,12)$. Growth factors are known to regulate cellular growth, differentiation, and function during various pathophysiological conditions and, during normal fetal and child growth $(13,14)$. Growth hormone and
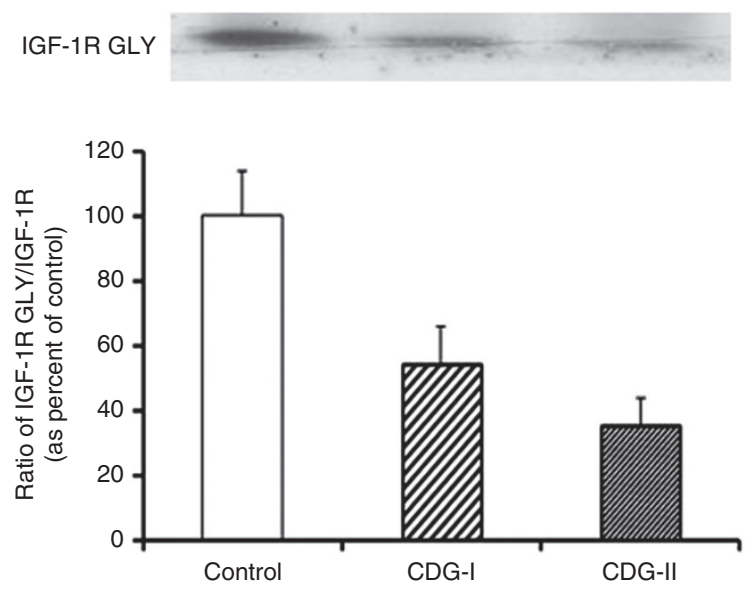

Figure 6. Detection of carbohydrate content of immune-precipitated insulin-like growth factor-1 (IGF-1)R protein from lymphocytes of control subjects and CDG patients. Data shown is mean \pm SD of six determinations. $P<0.01$ when CDG-I and CDG-II were compared with control.

IGF-1 are key regulatory peptides in muscle development, brain growth, myelination, development, and function of the nervous system $(15,16)$. PDGF has an established role in angiogenesis and neovascularization $(17,18)$. FGF participates in growth and function of several tissues and organs that range from organization of musculoskeletal system to formation of extracellular matrix and wound healing (19). Dysfunction of growth factor activity has been shown to affect the normal growth in children and development of various diseases and complex syndromes $(20,21)$. Impairment of mitogen (serum)-stimulated DNA synthesis as observed in lymphocytes of both CDG type-I and CDG type-II patients would indicate impedition of development in affected children. Serum is comprised of numerous growth promoting agents ranging from active metabolites to bioactive receptor-binding peptides that act solely or with other factors to stimulate cell growth. The observed selective impairment of IGF-1-induced synthesis of DNA in CDG patients in our study strongly suggests an association of growth factor dysfunction with pathogenic mechanisms for this group of inherited metabolic disorders. Due to the fact that IGF-1 is involved in a number of cellular activities at various developmental stages in children (14), our findings would strongly indicate that inhibition of growth-mediated activity may, at least in part, be responsible for some of the reported clinical characteristics of growth retardation in CDG patients. Significantly reduced levels of IGF-1 in plasma of CDG type-II patients further support the contention that insufficient activity of growth factor(s) may be a contributing factor in pathogenesis of this group of metabolic disorders, however, it is intriguing to observe that IGF-I levels were normal in CDG type-I patients. Cellular growth factor activities are not dependent only on the availability or levels of a growth factor but also require normal structure and function of its specific receptor. It was interesting to find that IGF-1 receptor protein was significantly reduced in lymphocytes of only CDG type-I patients but not in CDG type-II patients, further pointing out differences in mechanisms associated with dysfunction of IGF-1 in CDG patients. These findings were 
intriguing in light of our observation that IGF-1-induced DNA synthesis was impaired in lymphocytes of both CDG type-I and type-II patients and prompted investigations to further explore the mechanisms. Numerous growth factor receptors are complexly modified proteins and improper post-translational modifications of these proteins can lead to dysfunction of the growth factor activity despite normal levels of the growth factor and its receptor protein. IGF-1 receptor is a glycoprotein and requires proper post-translational glycosylation of its protein, like several other glycoproteins, for a normal function (22). Our finding that IGF-1 receptor had markedly reduced carbohydrate content in lymphocytes of all CDG patients unravelled the hypoglycosylation of the IGF-1 receptor in CDG. Hypoglycosylation of glycoproteins is a hallmark of the biochemical defect in CDG patients as several glycoproteins, such as transferrin and haptoglobin are well known to be deglycosylated due to inborn error in glycosyaltion enzymes and form a diagnostic tool for this group of inherited metabolic disorders. Hypoglycosylation of IGF-1 receptor, partly, explains the observed selective impairment of IGF-1-mediated DNA synthesis in lymphocytes of CDG patients, but strongly suggests that dysfunction of growth factor(s) might be an important factor contributing to pathogenesis of CDG. The fact that all CDG patients, in this study, had impaired mitogenic response to FBS and IGF-1 further underscores the common important feature of protein hypoglycosylation in CDG irrespective of the enzymatic defect. This study is limited to lymphocytes, an excellent noninvasive model for cell culture studies to delineate molecular mechanisms, but provides a strong inference to a role of growth factors in pathogenesis of CDG as the underlying biochemical defect of protein glycosylation in CDG patients is not limited only to lymphocytes but leads to hypoglycosylation of most glycoproteins in all tissues. Further, the selective inhibition of IGF-1-induced DNA synthesis, observed in this study, may only be limited to lymphocytes, as different cells and tissues are diversified in production of growth factors and expression of their receptors suggesting that growth factors other than IGF-1 may also have a role to play in pathogenesis of CDG. This study has provided some new insights into molecular mechanisms of CDG pathogenesis and unravels hypoglycosylation-mediated impairment of growth factor activities in CDG patients. Since IGF- 1 and its receptor are now reported to be involved in several other pathophysiological conditions such as cancer and atherogenesis (23), our findings might suggest a role for protein deglycosylation in pathogenic mechanisms and/or providing molecular tools for treatment of diseases other than CDG.

\section{METHODS}

\section{Materials}

Cell culture flasks, plates, and petri dishes were purchased from Falcon Becton Dickinson (Oxnard, CA). Cell culture media, RPMI 1640, Dulbecco's Modified Eagle's Medium (DMEM) and DMEMHam's F-12, were obtained from GIBCO (Grand Island, NY). Trypsin-Ethylenediaminetetraacetic acid (EDTA), FBS, penicillin/ Streptomycin, insulin-transferrin-selenium were procured from Sigma Chemical Company (St. Louis, MO). Bovine serum albumin, IGF-1, PDGF, and basic fibroblast growth factor (bFGF) were purchased from Calbiochem (La Jolla, CA). Carbohydrate staining and BrdU incorporation kits as well as all other chemicals and reagents were obtained from Sigma. Antibody against IGF-1 receptor was from Santa Cruz Biotechnology, Santa Cruz, CA.

\section{Subjects}

Blood samples from 9 CDG patients (6 patients type-I and 3 patients type-II) and, 12 healthy (age and sex matched) controls were collected according to the protocols set and approved by the Institutional Ethical Committee on use of Human Subjects in Research. Parental informed consent was obtained for each patient and control (MK 01/05). Molecular diagnosis could not be performed in this study and the CDG patients were broadly grouped as CDG-I and CDG-II based upon confirmed diagnosis by isoelectric focusing and immunoblot analysis of serum transferrin as described earlier (6). All patients and healthy controls included in this study were drug free and ranged from 8 mo to $9.2 \mathrm{y}$ in age.

\section{Cell Cultures}

Peripheral blood mononuclear cells were isolated from CDG patients and healthy control subjects using ficol-gradient centrifugation. Pure cultures of lymphocytes were established by culturing peripheral blood mononuclear cells for 5-7 d according to the modified method of Haller and co-workers (24) and maintained in growth medium (RPMI 1640 containing 10\% FBS, and penicillin and streptomycin, $5 \mathrm{U} / \mathrm{ml}$ and $5 \mu \mathrm{g} / \mathrm{ml}$ respectively) in a humidified atmosphere of $5 \%$ $\mathrm{CO}_{2}-95 \%$ air. Cells were cultured in $25 \mathrm{~mm}^{2}, 75 \mathrm{~mm}^{2}$, petri dishes, 6-well or 96-well plates depending upon the assay to be performed. Purity and yield of the isolated nonadherent lymphocytes were determined by the cell-surface staining technique using the fluorescein isothiocyanate-phycoerythrin-labeled anti-CD3 (Becton Dickinson) monoclonal antibodies.

\section{Treatment With Experimental Agents}

Lymphocyte cultures from CDG patients and healthy controls were grown in 6-well plates and 96-well plated, and treated for $24 \mathrm{~h}$ with various mitogens, which included FBS (5\%), PDGF (50 ng/ml), bFGF $(50 \mathrm{ng} / \mathrm{ml})$, insulin-like growth factor-1 (IGF-1, $50 \mathrm{ng} / \mathrm{ml})$, and insulin-transferrin-selenous acid $(5 \mu \mathrm{g} / \mathrm{ml})$. Before treating the cells with growth factors, cell cultures were growth arrested by washing twice with serum free RPMI culture medium and incubating in serum free medium for $48 \mathrm{~h}$. Quiescent cultures of lymphocytes were then treated with chosen concentrations of mitogens for $24 \mathrm{~h}$ to study DNA synthesis.

\section{Measurement of DNA Synthesis}

Cells cultures established in 96-well plates were used for experiments to study DNA synthesis. Following treatment of cell cultures with experimental agents (FBS or growth factors), BrdU incorporation into cells was measured as an index of DNA synthesis. BrdU was added $2 \mathrm{~h}$ after addition of growth factors to serum-starved cells and incorporation of BrdU into lymphocytic DNA was measured using Calbiochem kits.

\section{Western Blot Analysis}

Cell cultures were set up in six-well plates for western blot studies. Lymphocytes from CDG patients and control subjects were harvested and centrifuged into cell pellets for western blot analysis. Cell pellets were homogenized and solubilized in lysis buffer ( $\mathrm{pH}$ 7.6) that contained $50 \mathrm{mmol} / \mathrm{l}$ Tris-base, $5 \mathrm{mmol} / \mathrm{l}$ Ethyleneglycol-bis-tetraacetic acid (EGTA), $150 \mathrm{mmol} / \mathrm{l} \mathrm{NaCl}, 1 \%$ Triton X-100, $2 \mathrm{mmol} / \mathrm{l} \mathrm{Na}_{3} \mathrm{VO}_{4}$, $1 \mathrm{mmol} / \mathrm{l}$ Phenylmethylsulfonylflouride (PMSF), $10 \mathrm{mmol} / \mathrm{l}$ sodium molybdate, and cocktail of protease inhibitors. Cell lysates were centrifuged and supernatants were collected, and protein was estimated using Bio-Rad BCA protein assay (Hercules, CA). Cell lysate aliquots of lymphocytes from CDG patients and controls were subjected in equal amounts to sodium dodecyl sulfate-polyacrylamide gel electrophoresis. Proteins were transferred onto nitrocellulose membranes and probed with antibodies against IGF- 1 receptor and $\beta$-actin. Immunoreactive bands were quantified following detection with SuperSignal chemiluminescent substrate (Pierce, Cheshire, UK) and densitometric measurements. 


\section{Articles | Dhaunsi}

\section{Immunoprecipitation and Carbohydrate Staining of IGF-1R}

Cell lysates prepared from CDG patients and control subjects were mixed with $1 \mu \mathrm{g} / \mathrm{ml}$ of IGF-1 R antibody and $5 \mathrm{ml}$ of Protein G Plus Agarose. After a 24 -h incubation at $4{ }^{\circ} \mathrm{C}$ with constant shaking on a rocker, immunoprecipitates were collected by centrifugation in a microcentrifuge. Pellets were washed three times with PBSTDS (phosphate-buffered saline containing 1\% TritonX-100, sodium deoxycholate, and sodium dodecyl sulfate) and samples in equal amount of protein were subjected to sodium dodecyl sulfate-polyacrylamide gel electrophoresis. Gels were then directly subjected to glycoprotein detection using a Periodic Acid-Schiff-based kit or silver staining kit for detection of protein bands. Glycoprotein detection produced magenta colored bands with light pink or colorless background.

\section{Assay of Plasma IGF-1}

Total IGF-1 concentrations in plasma samples of all CDG patients and control subjects were measured using commercially available ELISA kits from Assay Design (Ann Arbor, MI).

\section{Statistical Analysis}

Data were analyzed for statistical significance using student's ' $t$ ' test by single comparison of mean values. Multiple comparisons were carried out by one-way ANOVA followed by Scheffe's test for post-hoc comparison of selected means. $P<0.05$ in favor of the null hypothesis was considered to indicate statistical significance.

\section{ACKNOWLEDGMENT}

Technical assistance of Nini Mathew is greatly acknowledged.

\section{STATEMENT OF FINANCIAL SUPPORT}

This study was supported by a research grant from Kuwait University (MK 01/05).

Disclosure: There is no conflict of interest.

\section{REFERENCES}

1. Jaeken J, Matthijs G. Congenital disorders of glycosylation: a rapidly expanding disease family. Annu Rev Genomics Hum Genet 2007;8:261-78.

2. Jaeken J. Congenital disorders of glycosylation. Ann N Y Acad Sci 2010;1214:190-8.

3. Woods AG, Woods CW, Snow TM. Congenital disorders of glycosylation. Adv Neonatal Care 2012;12:90-5.

4. Jaeken J. Congenital disorders of glycosylation (CDG): it's (nearly) all in it! J Inherit Metab Dis 2011;34:853-8.

5. Makhseed N, Dhaunsi G, Jaeken J. Distinct features of congenital disorder of glycosylation type IIx in Kuwait: a case report. J Child Neurol 2012;27:222-4.

6. Seta N, Barnier A, Hochedez F, Besnard MA, Durand G. Diagnostic value of Western blotting in carbohydrate-deficient glycoprotein syndrome. Clin Chim Acta 1996;254:131-40.
7. Carchon H, Van Schaftingen E, Matthijs G, Jaeken J. Carbohydrate-deficient glycoprotein syndrome type IA (phosphomannomutase-deficiency). Biochim Biophys Acta 1999;1455:155-65.

8. Baker J, Liu JP, Robertson EJ, Efstratiadis A. Role of insulin-like growth factors in embryonic and postnatal growth. Cell 1993;75:73-82.

9. Savage MO. Insulin-like growth factors, nutrition and growth. World Rev Nutr Diet 2013;106:52-9.

10. Yakar S, Rosen CJ, Beamer WG, et al. Circulating levels of IGF-1 directly regulate bone growth and density. J Clin Invest 2002;110:771-81.

11. Kolarich D, Lepenies B, Seeberger PH. Glycomics, glycoproteomics and the immune system. Curr Opin Chem Biol 2012;16:214-20.

12. Perdue JF. The role of somatomedin/insulin-like growth factors and their receptors in skeletal growth and fetal development: a mini-review. Prog Clin Biol Res 1985; 132B: 405-413.

13. Werner H, LeRoith D. Insulin and insulin-like growth factor receptors in the brain: physiological and pathological aspects. Eur Neuropsychopharmacol 2014;24:1947-53.

14. LeRoith D, Yakar S. Mechanisms of disease: metabolic effects of growth hormone and insulin-like growth factor 1. Nat Clin Pract Endocrinol Metab 2007;3:302-10.

15. Aberg ND, Brywe KG, Isgaard J. Aspects of growth hormone and insulinlike growth factor-I related to neuroprotection, regeneration, and functional plasticity in the adult brain. ScientificWorldJournal 2006;6:53-80.

16. Mohan S, Kesavan C. Role of insulin-like growth factor-1 in the regulation of skeletal growth. Curr Osteoporos Rep 2012;10:178-86.

17. Lee C, Zhang F, Tang Z, Liu Y, Li X. PDGF-C: a new performer in the neurovascular interplay. Trends Mol Med 2013;19:474-86.

18. Shah P, Keppler L, Rutkowski J. A review of platelet derived growth factor playing pivotal role in bone regeneration. J Oral Implantol 2014;40:330-40.

19. Itoh N, Ornitz DM. Fibroblast growth factors: from molecular evolution to roles in development, metabolism and disease. J Biochem 2011;149: 121-30.

20. Setia S, Sridhar MG. Changes in GH/IGF-1 axis in intrauterine growth retardation: consequences of fetal programming? Horm Metab Res 2009;41:791-8.

21. Gasperi M, Castellano AE. Growth hormone/insulin-like growth factor I axis in neurodegenerative diseases. J Endocrinol Invest 2010;33:587-91.

22. Siddle K. The insulin receptor and type I IGF receptor: comparison of structure and function. Prog Growth Factor Res 1992;4:301-20.

23. Mohanraj L, Oh Y. Targeting IGF-I, IGFBPs and IGF-I receptor system in cancer: the current and future in breast cancer therapy. Recent Pat Anticancer Drug Discov 2011;6:166-77.

24. Haller D, Blum S, Bode C, Hammes WP, Schiffrin EJ. Activation of human peripheral blood mononuclear cells by nonpathogenic bacteria in vitro: evidence of NK cells as primary targets. Infect Immun 2000;68: $752-9$. 